\title{
IDENTITIES FOR A PARAMETRIC WEYL ALGEBRA OVER A RING
}

\author{
ARTEM LOPATIN AND CARLOS ARTURO RODRIGUEZ PALMA
}

\begin{abstract}
In 2013 Benkart, Lopes and Ondrus introduced and studied in a series of papers the infinite-dimensional unital associative algebra $\mathrm{A}_{h}$ generated by elements $x, y$, which satisfy the relation $y x-x y=h$ for some $0 \neq h \in \mathbb{F}[x]$. We generalize this construction to $A_{h}(B)$ by working over the fixed $\mathbb{F}$-algebra $B$ instead of $\mathbb{F}$. We describe the polynomial identities for $A_{h}(B)$ over the infinite field $\mathbb{F}$ in case $h \in \mathrm{B}[x]$ satisfies certain restrictions.

Keywords: polynomial identities, matrix identities, Weyl algebra, Ore extensions, positive characteristic.

2020 MSC: 16R10; 16S32.
\end{abstract}

\section{INTRODUCTION}

Assume that $\mathbb{F}$ is a field of arbitrary characteristic $p=\operatorname{char} \mathbb{F} \geq 0$. All vector spaces and algebras are over $\mathbb{F}$ and all algebras are associative, unless otherwise is stated. For the fixed $\mathbb{F}$-algebra $\mathrm{B}$ with unity we write $\mathrm{B}\left\langle x_{1}, \ldots, x_{m}\right\rangle$ for the $\mathbb{F}$ algebra of non-commutative $\mathrm{B}$-polynomials in variables $x_{1}, \ldots, x_{m}$, i.e., $\mathrm{B}\left\langle x_{1}, \ldots, x_{m}\right\rangle$ is a free left (and a free right) B-module with the basis given by the set of all noncommutative monomials in $x_{1}, \ldots, x_{m}$, where we assume that $\beta x_{i}=x_{i} \beta$ for all $\beta \in \mathrm{B}$ and $1 \leq i \leq m$. The unity 1 of $\mathrm{B}\left\langle x_{1}, \ldots, x_{m}\right\rangle$ corresponds to the empty monomial. In case the variables are $x_{1}, x_{2}, \ldots$ the algebra of non-commutative $\mathrm{B}$ polynomials is denoted by $\mathrm{B}\langle X\rangle$. Similarly, we define the algebra of commutative B-polynomials $\mathrm{B}\left[x_{1}, \ldots, x_{m}\right]$ as a free left (and a free right) B-module with the basis given by the set of all monomials in $x_{1}^{i_{1}} \cdots x_{m}^{i_{m}}$ with $i_{1}, \ldots, i_{m} \geq 0$, where we assume that $\beta x_{i}=x_{i} \beta$ and $x_{i} x_{j}=x_{j} x_{i}$ for all $\beta \in \mathrm{B}$ and $1 \leq i, j \leq m$. Note that $\mathrm{B}\langle x\rangle=\mathrm{B}[x]$.

1.1. Parametric Weyl algebra $A_{h}(B)$ as the Ore extension. We study the polynomial identities for the following family of infinite-dimensional unital algebras $\mathrm{A}_{h}(\mathrm{~B})$, which are parametrized by a polynomial $h$ from the center of $\mathrm{B}[x]$ :

Definition 1.1. For $h \in Z(\mathrm{~B})[x]$, the parametric Weyl algebra $\mathrm{A}_{h}(\mathrm{~B})$ over the ring $\mathrm{B}$ is the unital associative algebra over $\mathbb{F}$ generated by $\mathrm{B}$ and letters $x, y$ commuting with $\mathrm{B}$ subject to the defining relation $y x=x y+h$ (equivalently, $[y, x]=h$, where $[y, x]=y x-x y$ ), i.e.,

$$
\mathrm{A}_{h}(\mathrm{~B})=\mathrm{B}\langle x, y\rangle / \operatorname{id}\{y x-x y-h\} .
$$

For short, we denote $A_{h}=A_{h}(\mathbb{F})$. The partial cases of the given construction are the Weyl algebra $A_{1}$, the polynomial algebra $A_{0}=\mathbb{F}[x, y]$, and the universal

The first author was supported by CNPq 313358/2017-6, FAEPEX 2054/19 and FAEPEX $2655 / 19$. 
enveloping algebra $\mathrm{A}_{x}$ of the two-dimensional nonabelian Lie algebra. For $h \in \mathbb{F}[x]$, the following isomorphism of $\mathbb{F}$-algebras holds:

$$
\mathrm{A}_{h}(\mathrm{~B}) \simeq \mathrm{B} \otimes_{\mathbb{F}} \mathrm{A}_{h} .
$$

Note that in general the isomorphism (1) does not hold because $A_{h}$ is not welldefined in case $h \notin \mathbb{F}[x]$. Given a polynomial $f=\eta_{d} x^{d}+\eta_{d-1} x^{d-1}+\cdots+\eta_{0}$ of $\mathrm{B}[x]$ with $d \geq 0$, we say that $\eta_{d}$ is the leading coefficient of $f$ and the product $\eta_{d} x^{d}$ is the leading term of $f$.

The algebra $A_{h}$ was introduced and studied by Benkart, Lopes, Ondrus $9,8,10$, as a natural object in the theory of Ore extensions. In particular, they determined automorphisms of $A_{h}$ over an arbitrary field $\mathbb{F}$ and the invariants of $A_{h}$ under the automorphisms, completely described the simple modules and derivations of $A_{h}$ over any field. Then Lopes and Solotar [20] described the Hochschild cohomology $\mathrm{HH}^{\bullet}\left(\mathrm{A}_{h}\right)$ over a field of arbitrary characteristic. Over an algebraically closed field of zero characteristic simple $A_{h}$-modules were independently classified by Bavula [5]. In recent preprints [6, [7] Bavula continued the study of the automorphism group of $\mathrm{A}_{h}$.

Let us recall that an Ore extension of $R$ (or, equivalently, a skew polynomial ring over $R$ ) $A=\mathrm{R}[y, \sigma, \delta]$ is given by a unital associative (not necessarily commutative) algebra $\mathrm{R}$ over a field $\mathbb{F}$, an $\mathbb{F}$-algebra endomorphism $\sigma: \mathrm{R} \rightarrow \mathrm{R}$, and a $\sigma$-derivation $\delta: \mathrm{R} \rightarrow \mathrm{R}$, i.e., $\delta$ is $\mathbb{F}$-linear map and $\delta(a b)=\delta(a) b+\sigma(a) \delta(b)$ for all $a, b \in \mathrm{R}$. Then $A=\mathrm{R}[y, \sigma, \delta]$ is the unital algebra generated by $y$ over $\mathrm{R}$ subject to the relation

$$
y a=\sigma(a) y+\delta(a) \quad \text { for all } a \in \mathrm{R} .
$$

Assume that $\mathrm{R}=\mathrm{B}[x], \sigma=\mathrm{id}_{\mathrm{R}}$ is the identity automorphism on $\mathrm{R}$, and $\delta: \mathrm{R} \rightarrow \mathrm{R}$ is given by $\delta(a)=a^{\prime} h$ for all $a \in \mathrm{R}$, where $a^{\prime}$ stands for the usual derivative of a B-polynomial $a$ with respect to the variable $x$. Since $h \in Z(\mathrm{R}), \delta$ is a derivation of R. Using the linearity of derivative and induction on the degree of $a \in \mathrm{B}[x]$ it is easy to see that

$$
[y, a]=a^{\prime} h \text { holds in } \mathrm{A}_{h}(\mathrm{~B}) \text { for all } a \in \mathrm{B}[x] .
$$

Hence $\mathrm{A}_{h}(\mathrm{~B})=\mathrm{R}[y, \sigma, \delta]$ is an Ore extension. The following lemma is a corollary of Observation 2.1 from [3] proven by Awami, Van den Bergh and Van Oystaeyen (see also Proposition 3.2 of [1] and Lemma 2.2 of [9]).

Lemma 1.2. Assume that $A=\mathrm{R}[y, \sigma, \delta]$ is an Ore extension of $\mathrm{R}=\mathbb{F}[x]$, where $\sigma$ is an automorphism of $\mathrm{R}$. Then $A$ is isomorphic to one of the following algebras:

- a quantum plane, i.e., $A \simeq \mathbb{F}\langle x, y\rangle / \operatorname{id}\{y x-q x y\}$ for some $q \in \mathbb{F}^{*}=\mathbb{F} \backslash\{0\}$;

- a quantized Weyl algebra, i.e., $A \simeq \mathbb{F}\langle x, y\rangle / \operatorname{id}\{y x-q x y-1\}$ for some $q \in$ $\mathbb{F}^{*}$;

- an algebra $\mathrm{A}_{h}$ for some $h \in \mathbb{F}[x]$.

Note that by Theorem 9.3 of [9] the algebra $A_{h}$ is not a generalized Weyl algebra over $\mathbb{F}[x]$ in the sense of Bavula [4] in case $h \notin \mathbb{F}$.

Since the algebra of B-polynomials $\mathrm{B}[x, y]$ is well studied, in what follows we assume that $h$ is non-zero. Moreover, we assume that the following restriction holds:

Convention 1.3. The leading coefficient of $h \in Z(\mathrm{~B})[x]$ is not a zero divisor. 
1.2. Polynomial identities. A polynomial identity for a unital $\mathbb{F}$-algebra $\mathcal{A}$ is an element $f\left(x_{1}, \ldots, x_{m}\right)$ of $\mathbb{F}\langle X\rangle$ such that $f\left(a_{1}, \ldots, a_{m}\right)=0$ in $\mathcal{A}$ for all $a_{1}, \ldots, a_{m} \in$ $\mathcal{A}$. The set $\operatorname{Id}(\mathcal{A})$ of all polynomial identities for $\mathcal{A}$ is a $\mathrm{T}$-ideal, i.e., $\operatorname{Id}(\mathcal{A})$ is an ideal of $\mathbb{F}\langle X\rangle$ such that $\phi(\operatorname{Id}(\mathcal{A})) \subset \operatorname{Id}(\mathcal{A})$ for every endomorphism $\phi$ of $\mathbb{F}\langle X\rangle$. An algebra that satisfies a nontrivial polynomial identity is called a PI-algebra. A Tideal $I$ of $\mathbb{F}\langle X\rangle$ generated by $f_{1}, \ldots, f_{k} \in \mathbb{F}\langle X\rangle$ is the minimal T-ideal of $\mathbb{F}\langle X\rangle$ that contains $f_{1}, \ldots, f_{k}$. We say that $f \in \mathbb{F}\langle X\rangle$ follows from $f_{1}, \ldots, f_{k}$ if $f \in I$. Given a monomial $w \in \mathbb{F}\left\langle x_{1}, \ldots, x_{m}\right\rangle$, we write $\operatorname{deg}_{x_{i}}(w)$ for the number of letters $x_{i}$ in $w$ and $\operatorname{mdeg}(w)$ for the multidegree $\left(\operatorname{deg}_{x_{1}}(w), \ldots, \operatorname{deg}_{x_{m}}(w)\right)$ of $w$. An element $f \in \mathbb{F}\langle X\rangle$ is called multihomogeneous if it is a linear combination of monomials of the same multidegree. We say that algebras $\mathcal{A}, \mathcal{B}$ are called PI-equivalent and write $\mathcal{A} \sim_{\text {PI }} \mathcal{B}$ if $\operatorname{Id}(\mathcal{A})=\operatorname{Id}(\mathcal{B})$.

Denote the $n^{\text {th }}$ Weyl algebra by

$$
\mathcal{A}_{n}=\mathbb{F}\left\langle x_{1}, \ldots, x_{n}, y_{1}, \ldots, y_{n}\right\rangle / I
$$

where the ideal $I$ is generated by $\left[y_{i}, x_{j}\right]=\delta_{i j},\left[x_{i}, x_{j}\right]=0,\left[y_{i}, y_{j}\right]=0$ for all $1 \leq i, j \leq n$. Note that $\mathrm{A}_{1}=\mathcal{A}_{1}$.

Assume that $p=0$. It is well-known that the algebra $\mathcal{A}_{n}$ does not have nontrivial polynomial identities. Nevertheless, some subspaces of $\mathcal{A}_{n}$ satisfy certain polynomial identities. Namely, denote by $\mathcal{A}_{n}^{(1,1)}$ the $\mathbb{F}$-span of $x_{i} y_{j}$ in $\mathcal{A}_{n}$ for all $1 \leq i, j \leq n$ and by $\mathcal{A}_{n}^{(-, r)}$ the $\mathbb{F}$-span of $a y_{j_{1}} \cdots y_{j_{r}}$ in $\mathcal{A}_{n}$ for all $1 \leq j_{1}, \ldots, j_{r} \leq n$ and $a \in \mathbb{F}\left[x_{1}, \ldots, x_{n}\right]$. Dzhumadil'daev [12, 13] studied the standard polynomial

$$
\operatorname{St}_{N}\left(t_{1}, \ldots, t_{N}\right)=\sum_{\sigma \in S_{N}}(-1)^{\sigma} t_{\sigma(1)} \cdots t_{\sigma(N)}
$$

over some subspaces of $\mathcal{A}_{n}$. Namely, he showed that

- $\mathrm{St}_{N}$ is a polynomial identity for $\mathcal{A}_{n}^{(-, 1)}$ in case $N \geq n^{2}+2 n$;

- $\mathrm{St}_{N}$ is not a polynomial identity for $\mathcal{A}_{n}^{(-, 1)}$ in case $N<n^{2}+2 n-1$;

- $\mathrm{St}_{N}$ is a polynomial identity for $\mathcal{A}_{1}^{(-, r)}$ if and only if $N>2 r$;

- the minimal degree of nontrivial identity in $\mathcal{A}_{1}^{(-, r)}$ is $2 r+1$.

Using graph-theoretic combinatorial approach Dzhumadil'daev and Yeliussizov [14 established that

- $\mathrm{St}_{2 n}$ is a polynomial identity for $\mathcal{A}_{n}^{(1,1)}$ if and only if $n=1,2,3$.

Note that the space $\mathcal{A}_{n}^{(-, 1)}$ together the multiplication given by the Lie bracket is the $n^{\text {th }}$ Witt algebra $W_{n}$, which is a simple infinitely dimensional Lie algebra. The polynomial identities for the Lie algebra $W_{n}$ were studied by Mishchenko [21, Razmyslov [22] and others. The well-known open conjecture claims that all polynomial identities for $W_{1}$ follow from the standard Lie identity

$$
\sum_{\sigma \in S_{4}}(-1)^{\sigma}\left[\left[\left[\left[t_{0}, t_{\sigma(1)}\right] t_{\sigma(2)}\right] t_{\sigma(3)}\right] t_{\sigma(4)}\right] .
$$

$\mathbb{Z}$-graded identities for $W_{1}$ were described by Freitas, Koshlukov and Krasilnikov [15].

1.3. Results. In Theorem 4.9 we prove that over an infinite field $\mathbb{F}$ of positive characteristic $p$ the algebra $\mathrm{A}_{h}(\mathrm{~B})$ is PI-equivalent to the algebra of $p \times p$ matrices over $\mathrm{B}$ in case $h(\alpha)$ is not a zero divisor for some $\alpha \in Z(\mathrm{~B})$. On the other hand, over a finite field the similar result does not hold in case $B=\mathbb{F}$ (see Theorem 5.1). 
As about the case of zero characteristic, in Theorem 3.2 we prove that similarly to $A_{1}$, the algebra $A_{h}(B)$ does not have nontrivial polynomial identities.

In Section [6 we consider the algebra $\mathrm{A}_{h}(\mathrm{~B})=\mathrm{A}_{\zeta}\left(\mathbb{F}^{2}\right)$ such that $h=\zeta$ does not satisfy Convention 1.3 and the statements of Theorems 3.2 and 4.9 do not hold for $A_{\zeta}\left(\mathbb{F}^{2}\right)$. We describe polynomial identities for $A_{\zeta}\left(\mathbb{F}^{2}\right)$ and compare them with the polynomial identities for the Grassmann unital algebra of finite rank.

\section{Properties of $\mathrm{A}_{h}(\mathrm{~B})$}

Many properties of an Ore extension $A=\mathrm{R}[y, \sigma, \delta]$ are inherited from an underlying algebra R. Namely, it is well-known that when $\sigma$ is an automorphism, then:

- $A$ is a free right and a free left R-module with the basis $\left\{y^{i} \mid i \geq 0\right\}$ (see Proposition 2.3 of $[19]$ );

- in case $R$ is left (right, respectively) noetherian we have that $A$ is left (right, respectively) noetherian (see Theorem 2.6 of [19]);

- in case $\mathrm{R}$ is a domain we have that $A$ is a domain (see Exercise $2 \mathrm{O}$ of [19]).

In case $\mathrm{B}=\mathbb{F}$ the algebra $\mathrm{A}_{h}=\mathrm{A}_{h}(\mathrm{~B})$ is a noetherian domain, but in general case $\mathrm{A}_{h}(\mathrm{~B})$ lacks these properties, since $\mathrm{B} \subset \mathrm{A}_{h}(\mathrm{~B})$ (see also Example 2.6 below).

In order to distinguish the generators for the algebras $A_{h}(B)$ and $A_{1}(B)$, we will use the following

Convention 2.1. The generators of $\mathrm{A}_{h}(\mathrm{~B})$ are denoted by $x, \widehat{y}, 1$ and the generators of $\mathrm{A}_{1}(\mathrm{~B})$ are denoted by $x, y, 1$.

Lemma 2.2. The sets $\left\{x^{i} \widehat{y}^{j} \mid i, j \geq 0\right\}$ and $\left\{\widehat{y}^{j} x^{i} \mid i, j \geq 0\right\}$ are $\mathrm{B}$-bases for $\mathrm{A}_{h}(B)$.

Proof. Obviously, $\mathrm{A}_{h}(\mathrm{~B})$ is the B-span of each of the sets from the lemma. On the other hand, $B$-linear independence of these sets follows from the fact that $A_{h}(B)$ is a free right and a free left $\mathrm{B}[x]$-module with the basis $\left\{\widehat{y}^{i} \mid i \geq 0\right\}$.

Introduce the following lexicographical order on $\mathbb{Z}^{2}:(i, j)<(r, s)$ in case $j<s$ or $j=s, i<r$. Denote the multidegree of a monomial $w=x^{i} \widehat{y}^{j}$ of $\mathrm{A}_{h}(\mathrm{~B})$ by $\operatorname{mdeg}(w)=(i, j)$. Given an arbitrary element $a=\sum_{i, j \geq 0} \beta_{i j} x^{i} \widehat{y}^{j}$ of $\mathrm{A}_{h}(\mathrm{~B})$, where only finitely many $\beta_{i j} \in \mathrm{B}$ are non-zero, define its multidegree mdeg $(a)=\left(d_{x}, d_{y}\right)$ as the maximal multidegree of its momomials, i.e. as the maximal element of the set $\left\{(i, j) \mid \beta_{i j} \neq 0\right\}$. By Lemma 2.2 the multidegree is well-defined. As above, the coefficient $\beta_{d_{x}, d_{y}}$ is called the leading coefficient of $a$ and the product $\beta_{d_{x}, d_{y}} x^{d_{x}} \widehat{y}^{d_{y}}$ is called the leading term of $a$. In case $a \in \mathrm{B}$ we set $\operatorname{mdeg}(a)=(0,0)$ and the leading coefficient as well as the leading term of $a$ is $a$.

Lemma 2.3. Assume $i, j, r, s \geq 0$. Then

(a) the leading term of $x^{i} \widehat{y}^{j} \cdot x^{r} \widehat{y}^{s}$ is $x^{i+r} \widehat{y}^{j+s}$;

(b) in case $h \in \mathrm{B}$ we have

$$
x^{i} \widehat{y}^{j} \cdot x^{r} \widehat{y}^{s}=\sum_{k=0}^{\min \{j, r\}} k !\left(\begin{array}{l}
j \\
k
\end{array}\right)\left(\begin{array}{l}
r \\
k
\end{array}\right) x^{i+r-k} h^{k} \widehat{y}^{j+s-k} .
$$


Proof. Recall that $\delta(a)=a^{\prime} h$ for each $a \in \mathrm{B}[x]$. Since $[\widehat{y}, a]=\delta(a)$, the induction on $j$ implies that

$$
\widehat{y}^{j} x^{r}=\sum_{k=0}^{j}\left(\begin{array}{l}
j \\
k
\end{array}\right) \delta^{k}\left(x^{r}\right) \widehat{y}^{j-k}
$$

(cf. Lemma 5.2 of 9 ). Taking $k=0$ in equality (3), we obtain that the leading term of $\widehat{y}^{j} x^{r}$ is $x^{r} \widehat{y}^{j}$. Similarly we conclude the proof of part (a). Part (b) follows from (3) and

$$
\delta^{k}\left(x^{r}\right)=\left\{\begin{array}{cl}
\frac{r !}{(r-k) !} x^{r-k} h^{k}, & \text { if } k \leq r \\
0, & \text { if } k>r
\end{array} .\right.
$$

Lemma 2.4. If the leading coefficient of one of non-zero elements $a, b \in \mathrm{A}_{h}(\mathrm{~B})$ is not a zero divisor, then $\operatorname{mdeg}(a b)=\operatorname{mdeg}(a)+\operatorname{mdeg}(b)$. In particular, ab is not zero.

Proof. Consider $a=\sum_{i=1}^{m} \beta_{i} x^{r_{i}} \widehat{y}^{s_{i}}$ and $b=\sum_{j=1}^{n} \gamma_{j} x^{k_{j}} \widehat{y}^{l_{j}}$ for $m, n \geq 1$ and nonzero $\beta_{i}, \gamma_{j} \in \mathrm{B}$, where we assume that elements of each of the sets $\left\{\left(r_{i}, s_{i}\right) \mid 1 \leq i \leq\right.$ $m\}$ and $\left\{\left(k_{j}, l_{j}\right) \mid 1 \leq j \leq n\right\}$ are pairwise different. Assume that $\operatorname{mdeg}(a)=\left(r_{1}, s_{1}\right)$ and $\operatorname{mdeg}(b)=\left(k_{1}, l_{1}\right)$. Part (a) of Lemma 2.3 implies that

$\operatorname{mdeg}\left(x^{r_{1}} \widehat{y}^{s_{1}} x^{k_{1}} \widehat{y}^{l_{1}}\right)=\left(r_{1}+k_{1}, s_{1}+l_{1}\right)$ and $\operatorname{mdeg}\left(x^{r_{i}} \widehat{y}^{s_{i}} x^{k_{j}} \widehat{y}^{l_{j}}\right)<\left(r_{1}+k_{1}, s_{1}+l_{1}\right)$ if $(i, j) \neq(1,1)$. Since $\beta_{1} \gamma_{1} \neq 0$, we obtain $\operatorname{mdeg}(a b)=\left(r_{1}+k_{1}, s_{1}+l_{1}\right)$ and the proof is concluded.

\section{Lemma 2.5.}

(a) The B-linear homomorphism of $\mathbb{F}$-algebras $\psi: \mathrm{A}_{h}(\mathrm{~B}) \rightarrow \mathrm{A}_{1}(\mathrm{~B})$, defined by

$$
1 \rightarrow 1, \quad x \rightarrow x, \quad \widehat{y} \rightarrow y h,
$$

is an embedding $\mathrm{A}_{h}(\mathrm{~B}) \subset \mathrm{A}_{1}(\mathrm{~B})$.

(b) $\left\{x^{i} h^{j} y^{j} \mid i, j \geq 0\right\}$ and $\left\{y^{j} h^{j} x^{i} \mid i, j \geq 0\right\}$ are $\mathrm{B}$-bases for $\mathrm{A}_{h}(\mathrm{~B}) \subset \mathrm{A}_{1}(\mathrm{~B})$.

Proof. (a) Since $\psi([\widehat{y}, x]-h)=([y, x]-1) h=0$ in $\mathrm{A}_{1}(\mathrm{~B})$, the homomorphism $\psi$ is well-defined. Assume that $\psi$ is not an embedding, i.e., there exists non-zero finite sum $a=\sum_{i, j \geq 0} \beta_{i j} x^{i} \widehat{y}^{j}$ with $\beta_{i j} \in \mathrm{B}$ such that

$$
\psi(a)=\sum_{i, j \geq 0} \beta_{i j} x^{i}(y h)^{j}=0 \quad \text { in } \quad \mathrm{A}_{1}(\mathrm{~B}) .
$$

Denote $\operatorname{mdeg}(a)=(r, s)$. If $(r, s)=(0,0)$, then $a \in \mathrm{B}$ and $\psi(a)=a$ is not zero; a contradiction. Therefore, $(r, s) \neq(0,0)$. Since $\operatorname{mdeg}\left(x^{i}(y h)^{j}\right)=(i+j \operatorname{deg}(h), j)$ by Lemma 2.4 and Convention 1.3, we obtain that $\operatorname{mdeg}(\psi(a))=(r+s \operatorname{deg}(h), s)$ is not zero; a contradiction.

(b) Since $h$ lies in the center of B $[x]$, repeating the proof of Lemma 3.4 from [9] for $\mathrm{A}_{h}(\mathrm{~B})$ we can see that

$$
\mathrm{A}_{h}(\mathrm{~B})=\bigoplus_{j \geq 0} \mathrm{~B}[x] h^{j} y^{j}=\bigoplus_{j \geq 0} y^{j} h^{j} \mathrm{~B}[x] .
$$

Similarly to part (a), we conclude the proof by the reasoning with multidegree. 
Example 2.6. Assume $B$ is the $\mathbb{F}$-algebra of double numbers, i.e., $B$ has an $\mathbb{F}$-basis $\{1, \zeta\}$ with $\zeta^{2}=0$. Then the ideal $I=\mathbb{F}$-span $\left\{\zeta x^{i} \widehat{y}^{j} \mid i, j \geq 0\right\}$ is a proper nilpotent ideal of $\mathrm{A}_{h}(\mathrm{~B})$. In particular, the algebra $\mathrm{A}_{h}(\mathrm{~B})$ is not semi-prime.

\section{3. $\mathrm{A}_{h}(\mathrm{~B})$ AS THE ALGEBRA OF DifFerential operators}

Denote by $\operatorname{Map}(\mathrm{B}[z])$ the algebra of all $\mathbb{F}$-linear maps over $\mathrm{B}[z]$ with respect to composition. Assume that $\mathfrak{D}_{h}(\mathrm{~B}[z])$ is the subalgebra of $\operatorname{Map}(\mathrm{B}[z])$ generated by the following maps: the multiplication $\beta$ Id by an element $\beta$ of B, i.e., $(\beta \operatorname{Id})(f)=\beta f$, the multiplication $\chi$ by $z$, i.e., $\chi(f)=z f$, and the derivation $\delta$ given by $\delta(f)=$ $f^{\prime} h(z)$ for all $f \in \mathrm{B}[z]$. Note that $\delta=h(\chi) \partial$, where $\partial$ stands for the operator of the usual derivative. Obviously, maps $\chi, h(\chi)$ and $\partial$ are B-linear. For short, we write $\chi^{0}$ for Id.

\section{Proposition 3.1.}

(a) $\left\{\chi^{i} h(\chi)^{j} \partial^{j} \mid i, j \geq 0\right\}$ is an $\mathrm{B}$-basis for $\mathfrak{D}_{h}(\mathrm{~B}[z])$ in case $p=0$.

(b) $\left\{\chi^{i} h(\chi)^{j} \partial^{j} \mid 0 \leq i, 0 \leq j<p\right\}$ is an $\mathrm{B}$-basis for $\mathfrak{D}_{h}(\mathrm{~B}[z])$ in case $p>0$.

(c) $\mathrm{A}_{h}(\mathrm{~B}) / \operatorname{id}\left\{h^{p} y^{p}\right\} \simeq \mathfrak{D}_{h}(\mathrm{~B}[z])$ for each $p \geq 0$.

Proof. Consider the B-linear homomorphism of $\mathbb{F}$-algebras $\Phi: \mathrm{A}_{1}(\mathrm{~B}) \rightarrow \operatorname{Map}(\mathrm{B}[z])$ given by $1 \rightarrow \mathrm{Id}, x \rightarrow \chi, y \rightarrow \partial$. Since $\Phi([y, x]-1)(f)=(\partial \chi-\chi \partial-\mathrm{Id})(f)=$ $f+z f^{\prime}-z f^{\prime}-f=0$ for all $f \in \mathrm{B}[z]$, the map $\Phi$ is a well-defined. Applying $\Phi$ to parts (a) and (b) of Lemma 2.5 we obtain that $\mathfrak{D}_{h}(\mathrm{~B}[z])=\Phi\left(\mathrm{A}_{h}(\mathrm{~B})\right)$ is an B-span of $\left\{\chi^{i} h(\chi)^{j} \partial^{j} \mid i, j \geq 0\right\}$.

Let $p=0$. Assume that some non-zero finite sum $\pi=\sum_{i, j>0} \beta_{i j} \chi^{i} h(\chi)^{j} \partial^{j}$ with $\beta_{i j} \in \mathrm{B}$ belongs to the kernel of $\Phi$. Denote by $j_{0}$ the minimal $j \geq 0$ with $\beta_{i j} \neq 0$ for some $i$ and denote by $i_{0}$ the maximal $i$ with $\beta_{i j_{0}} \neq 0$. Then $\pi\left(z^{j_{0}}\right)=j_{0} ! h(z)^{j_{0}} \sum_{0 \leq i \leq i_{0}} \beta_{i, j_{0}} z^{i}=0$ in $\mathrm{B}[z]$. Thus Convention 1.3 together with $j_{0} ! \beta_{i_{0}, j_{0}} \neq 0$ implies a contradiction. Part (a) is proven.

Assume that $p>0$ and some non-trivial finite sum

$$
\pi=\sum_{0 \leq i, 0 \leq j<p} \beta_{i j} \chi^{i} h(\chi)^{j} \partial^{j}
$$

with $\beta_{i j} \in \mathrm{B}$ belongs to the kernel of $\Phi$. As above, we obtain a contradiction. Since $\partial^{p}=0$, we conclude the proof of part (b). (c).

Parts (a) and (b) together with part (b) of Lemma 2.5 conclude the proof of part

Theorem 3.2. In case $p=0$ the algebra $\mathrm{A}_{h}(\mathrm{~B})$ does not have nontrivial polynomial identities.

Proof. Assume that $\mathbb{F}$-algebra $\mathrm{A}_{h}(\mathrm{~B})$ has a nontrivial polynomial identity. Since $p=0$, there exists $N>0$ such that $\mathrm{A}_{h}(\mathrm{~B})$ satisfies a nontrivial multilinear identity $f\left(x_{1}, \ldots, x_{N}\right)=\sum_{\sigma \in S_{N}} \alpha_{\sigma} x_{\sigma(1)} \cdots x_{\sigma(N)}$ with $\alpha_{\sigma} \in \mathbb{F}$. Moreover, we can assume that $\alpha_{\mathrm{Id}} \neq 0$ for the identity permutation Id. Given $j>0$, we write $F_{j}$ for a B-linear map $\chi^{2 j} h(\chi)^{j} \partial^{j}$ from $\mathfrak{D}_{h}(\mathrm{~B}[z])$. Denote by $d \geq 0$ the degree of $h$ and we write $\eta$ for the leading coefficient of $h$. Recall that $\eta$ is not a zero divisor by Convention 1.3. Note that

$$
F_{j}\left(z^{m}\right)=\left\{\begin{array}{cc}
0, & \text { in case } m<j \\
\frac{m !}{(m-j) !} z^{m+j} h(z)^{j}, & \text { in case } m \geq j .
\end{array}\right.
$$


In particular, $\operatorname{deg}\left(F_{j}\left(z^{j}\right)\right)=j(d+2)$ and the leading coefficient of $F_{j}\left(z^{j}\right)$ is $j ! \eta^{j}$, which is not a zero divisor.

By parts (a) and (c) of Proposition 3.1, the equality $f\left(F_{j_{1}}, \ldots, F_{j_{N}}\right)=0$ holds in $\mathfrak{D}_{h}(\mathrm{~B}[z])$ for all $j_{1}, \ldots, j_{N}>0$. Consider $j_{k}=(d+2)^{N-k}$ for all $0 \leq k \leq N$. Note that $1=j_{N}<j_{N-1}<\cdots<j_{0}$. We claim that for any $\sigma \in S_{N}$ we have

$$
F_{j_{\sigma(1)}} \circ \cdots \circ F_{j_{\sigma(N)}}(z) \neq 0 \text { if and only if } \sigma=\mathrm{Id} \text {. }
$$

The leading term of $F_{j_{1}} \circ \cdots \circ F_{j_{N}}(z)$ is $j_{1} ! \cdots j_{N} ! \eta^{j_{1}+\cdots+j_{N}} z^{j_{0}}$.

Let us prove these claims. Assume that $F_{j_{\sigma(1)}} \circ \cdots \circ F_{j_{\sigma(N)}}(z) \neq 0$ for some $\sigma \in S_{N}$.

Since $F_{j_{\sigma(N)}}(z) \neq 0$, then equality (4) implies that $\sigma(N)=N, j_{\sigma(N)}=1$, $\operatorname{deg}\left(F_{j_{\sigma(N)}}(z)\right)=d+2=j_{N-1}$ and the leading coefficient of $F_{j_{\sigma(N)}}(z)$ is $\eta$, which is not a zero divisor.

Similarly, assume that for $1 \leq l<N$ with $\sigma(l) \leq l$ the inequality $F_{j_{\sigma(l)}}(g) \neq 0$ holds for some $g \in \mathrm{B}[z]$ with the leading term $j_{l+1} ! \cdots j_{N} ! \eta^{j_{l+1}+\cdots+j_{N}} z^{j_{l}}$. Then equality (44) implies that $\sigma(l)=l$ and $\operatorname{deg}\left(F_{j_{\sigma(l)}}(g)\right)=j_{l-1}$. Moreover, the leading term of $F_{j_{\sigma(l)}}(g)$ is $j_{l} ! \cdots j_{N} ! \eta^{j_{l}+\cdots+j_{N}} z^{j_{l-1}}$. Consequently applying this reasoning to $l=N-1, l=N-2, \ldots, l=1$, we conclude the proof of claims (5) and (6).

Claims (5) and (6) imply that $0=f\left(F_{1}, \ldots, F_{N}\right)(z)=\alpha_{\mathrm{Id}} F_{j_{1}} \circ \cdots \circ F_{j_{N}}(z) \neq 0$ by Convention 1.3. a contradiction.

\section{Polynomial Identities for $\mathrm{A}_{h}(\mathrm{~B})$ in positive Characteristic}

We write $M_{n}=M_{n}(\mathbb{F})$ for the algebra of all $n \times n$ matrices over $\mathbb{F}$ and denote by $\widetilde{M}_{n}$ the algebra of all $n \times n$ matrices over $\mathrm{B}[x, y]$. Denote by $I_{n}$ the identity $n \times n$ matrix and by $E_{i j} \in M_{n}$ the matrix such that the $(i, j)^{\text {th }}$ entry is equal to one and the rest of entries are zeros. Consider the properties of the next two matrices of $M_{p}$ :

$$
A_{0}=\sum_{i=1}^{p-1} E_{i+1, i} \quad \text { and } \quad B_{0}=\sum_{i=1}^{p-1} i \cdot E_{i, i+1}
$$

\section{Lemma 4.1.}

(a) For all $0 \leq k<p$ we have that

$$
A_{0}^{k}=\sum_{i=1}^{p-k} E_{k+i, i} \quad \text { and } \quad B_{0}^{k}=\sum_{i=1}^{p-k} \frac{(k+i-1) !}{(i-1) !} E_{i, k+i},
$$

where $A_{0}^{0}$ and $B_{0}^{0}$ stand for $I_{p}$.

(b) $B_{0} A_{0}-A_{0} B_{0}=I_{p}$.

Proof. The formula for $A_{0}^{k}$ is trivial. We prove the formula for $B_{0}^{k}$ by induction on $k$. For $k=1$ the claim holds. Assume that the claim is valid for some $k<p-1$. Then

$$
B_{0}^{k+1}=\left(\sum_{i=1}^{p-k} \frac{(k+i-1) !}{(i-1) !} E_{i, k+i}\right)\left(\sum_{r=1}^{p-1} r \cdot E_{r, r+1}\right)=\sum_{i=1}^{p-(k+1)} \frac{(i+k) !}{(i-1) !} E_{i, k+1+i}
$$

and the required is proven. Part (b) is straightforward. 
Define the B-linear homomorphism $\varphi: \mathrm{B}\langle x, y\rangle \rightarrow \widetilde{M}_{p}$ of algebras by

$$
x \mapsto A, \quad y \mapsto B, \quad 1 \mapsto I_{p},
$$

where $A=x I_{p}+A_{0}$ and $B=y I_{p}+B_{0}$. Since $\beta A=A \beta$ and $\beta B=B \beta$ for each $\beta \in B$, the homomorphism $\varphi$ is well-defined.

Lemma 4.2. The homomorphism $\varphi$ induces the injective B-linear homomorphism $\bar{\varphi}: \mathrm{A}_{1}(\mathrm{~B}) \rightarrow \widetilde{M}_{p}$. In particular, the restriction of $\bar{\varphi}$ to $\mathrm{A}_{h}(\mathrm{~B}) \subset \mathrm{A}_{1}(\mathrm{~B})$ is the injective $\mathrm{B}$-linear homomorphism $\mathrm{A}_{h}(\mathrm{~B}) \rightarrow \widetilde{M}_{p}$.

Proof. By part (b) of Lemma 4.1 we have that $\varphi(y x-x y-1)=B A-A B-I_{p}=0$. Therefore, $\varphi$ induces a B-linear homomorphism $\bar{\varphi}: \mathrm{A}_{1}(\mathrm{~B}) \rightarrow \widetilde{M}_{p}$ of algebras.

Assume that there exists a nonzero $a \in \mathrm{A}_{1}(\mathrm{~B})$ such that $\bar{\varphi}(a)=0$. Since $\left\{x^{i} y^{j} \mid i, j \geq 0\right\}$ is an B-basis for $\mathrm{A}_{1}(\mathrm{~B})$ by Lemma 2.2. we have $a=\sum_{i, j \geq 0} \beta_{i j} x^{i} y^{j}$ for a finite sum with $\beta_{i j} \in \mathrm{B}$. Thus $0=\bar{\varphi}(a)=\sum_{i, j \geq 0} \beta_{i j} A^{i} B^{j}$. The equalities

$$
A^{i}=\left(\begin{array}{cccc}
x^{i} & 0 & \cdots & 0 \\
* & * & \cdots & * \\
\vdots & \vdots & & \vdots \\
* & * & \cdots & *
\end{array}\right) \text { and } B^{j}=\left(\begin{array}{cccc}
y^{j} & * & \cdots & * \\
0 & * & \cdots & * \\
\vdots & \vdots & & \vdots \\
0 & * & \cdots & *
\end{array}\right)
$$

imply that the $(1,1)^{\text {th }}$ entry of $A^{i} B^{j}$ is $\left(A^{i} B^{j}\right)_{1,1}=x^{i} y^{j}$. Hence $0=(\varphi(a))_{1,1}=$ $\sum_{i, j \geq 0} \beta_{i j} x^{i} y^{j}$ in $\mathrm{B}[x, y]$. Hence $\beta_{i j}=0$ for all $i, j \geq 0$ and $a=0$; a contradiction. Therefore $\bar{\varphi}$ is injective.

Given $1 \leq i, j \leq p$ and $k \geq 1$, we write $z_{i j}(k)$ for $x_{i+p(j-1)+p^{2}(k-1)} \in \mathbb{F}\langle X\rangle$. The generic $p \times p$ matrix $X_{k}$ with non-commutative elements is the matrix $X_{k}=$ $\left(z_{i j}(k)\right)_{1 \leq i, j \leq p}$.

Corollary 4.3. $\operatorname{Id}\left(M_{p}(\mathrm{~B})\right) \subset \operatorname{Id}\left(\mathrm{A}_{h}(\mathrm{~B})\right)$, if the field $\mathbb{F}$ is infinite.

Proof. Lemma 4.2 implies that $\operatorname{Id}\left(\widetilde{M}_{p}\right) \subset \operatorname{Id}\left(\mathrm{A}_{h}(\mathrm{~B})\right)$.

Since $B \subset B[x, y]$, we have $\operatorname{Id}\left(\widetilde{M}_{p}\right) \subset \operatorname{Id}\left(M_{p}(\mathrm{~B})\right)$. On the other hand, assume that $f=f\left(x_{1}, \ldots, x_{n}\right)$ is a polynomial identity for $M_{p}(\mathrm{~B})$. Then $f\left(X_{1}, \ldots, X_{n}\right)=$ $\left(f_{i j}\right)_{1 \leq i, j \leq n}$ for some $f_{i j} \in \mathbb{F}\langle X\rangle$ with $f_{i j} \in \operatorname{Id}(\mathrm{B})$. It is well-known that for an infinite field $\mathbb{F}$ and a commutative unital $\mathbb{F}$-algebra $\mathcal{C}$ the polynomial identities for a unital $\mathbb{F}$-algebra $\mathcal{B}$ and $\mathcal{C} \otimes_{\mathbb{F}} \mathcal{B}$ are the same (for example, see Lemma 1.4.2 of [18]). Since $\mathrm{B}[x, y]=\mathbb{F}[x, y] \otimes_{\mathbb{F}} \mathrm{B}$, we obtain $f_{i j} \in \operatorname{Id}(\mathrm{B}[x, y])$ and $f \in \operatorname{Id}\left(\widetilde{M}_{p}\right)$. The required is proven.

For each $\alpha \in Z(\mathrm{~B})$ consider the evaluation B-linear homomorphism $\epsilon_{\alpha}: \mathrm{B}[x, y] \rightarrow$ $B$ of unital $\mathbb{F}$-algebras defined by

$$
x \mapsto \alpha, \quad y \mapsto 0
$$

and extend it to the evaluation homomorphism $\varepsilon_{\alpha}: \widetilde{M}_{p} \rightarrow M_{p}(\mathrm{~B})$. Since $\mathrm{A}_{h}(\mathrm{~B})$ is a subalgebra of $\widetilde{M}_{p}$ by means of embedding $\bar{\varphi}$ (see Lemma 4.2), we can consider the images of $x, \widehat{y} \in \mathrm{A}_{h}(\mathrm{~B})$ in $M_{p}(\mathrm{~B})$, which we denote by $C_{\alpha}$ and $D_{\alpha}$, respectively:

$$
\begin{array}{rlrl}
C_{\alpha}=\varepsilon_{\alpha}(\bar{\varphi}(x)) & =\varepsilon_{\alpha}(A) & & =\alpha I_{p}+A_{0}, \\
D_{\alpha}=\varepsilon_{\alpha}(\bar{\varphi}(\widehat{y})) & =\varepsilon_{\alpha}(B h(A)) & =B_{0} \varepsilon_{\alpha}(h(A)) .
\end{array}
$$


Obviously, $\beta C_{\alpha}=C_{\alpha} \beta$ and $\beta D_{\alpha}=D_{\alpha} \beta$ for each $\beta \in B$. To obtain the explicit description of the matrix $D_{\alpha}$ we calculate $h(A)$. For $r \geq 1$ denote the $r^{\text {th }}$ derivative of $h \in \mathrm{B}[x]$ by $h^{(r)}=\frac{d^{r} h}{d x^{r}}$ and write $h^{(0)}$ for $h$. Note that $h^{(r)}(\alpha)$ lies in the center of $\mathrm{B}$.

\section{Lemma 4.4.}

$$
h(A)=\sum_{i=1}^{p} \sum_{j=1}^{i} \frac{1}{(i-j) !} h^{(i-j)} E_{i j} .
$$

Proof. We start with the case of $h=x^{k} \in \mathrm{B}[x]$ for some $k \geq 0$. Obviously, the claim of the lemma holds for $h=1$. Therefore, we assume that $k \geq 1$. Since $A_{0}^{r}=0$ for all $r \geq p$, we have

$$
h(A)=A^{k}=\left(x I_{p}+A_{0}\right)^{k}=\sum_{r=0}^{\min \{k, p-1\}}\left(\begin{array}{l}
k \\
r
\end{array}\right) x^{k-r} A_{0}^{r} .
$$

Part (a) of Lemma 4.1 implies

$$
h(A)=\sum_{r=0}^{\min \{k, p-1\}}\left(\begin{array}{l}
k \\
r
\end{array}\right) x^{k-r}\left(\sum_{i=1}^{p-r} E_{r+i, i}\right) .
$$

Regrouping the terms we obtain

$$
h(A)=\sum_{i=1}^{p} \sum_{j=\max \{1, i-k\}}^{i}\left(\begin{array}{c}
k \\
i-j
\end{array}\right) x^{k-(i-j)} E_{i j} .
$$

Note that for $0 \leq r<p$ we can rewrite

$$
\frac{1}{r !} h^{(r)}=\left\{\begin{array}{rl}
\left(\begin{array}{l}
k \\
r
\end{array}\right) x^{k-r}, & \text { if } r \leq k \\
0, & \text { otherwise }
\end{array},\right.
$$

where $r$ ! is not zero in $\mathbb{F}$. Hence equality (7) implies that the claim holds for $h=x^{k}$.

The general case follows from the proven partial case and the B-linearity of derivatives.

Lemma 4.4 together with the definition of $D_{\alpha}$ immediately implies the next corollary.

\section{Corollary 4.5.}

$$
D_{\alpha}=\sum_{i=1}^{p-1} \sum_{j=1}^{i+1} \frac{i}{(i-j+1) !} h^{(i-j+1)}(\alpha) E_{i j} .
$$

For short, denote the $(i, j)^{\text {th }}$ entry of $D_{\alpha}$ by $\xi_{i, j} \in Z(\mathrm{~B})$ and for all $1 \leq k<p$ define

$$
D_{\alpha, k}=D_{\alpha}-\sum_{r=0}^{k-1} \xi_{k, k-r} A_{0}^{r}=D_{\alpha}-\sum_{r=0}^{k-1} \sum_{i=1}^{p-r} \xi_{k, k-r} E_{r+i, i} .
$$


We apply the following technical lemma in the proof of key Proposition 4.7 (see below).

Lemma 4.6. For all $1 \leq r \leq p$ and $1 \leq k<p$ we have

$$
E_{r k} D_{\alpha, k}=k h(\alpha) E_{r, k+1} \text {. }
$$

Proof. We have

$$
\begin{aligned}
E_{r k} D_{\alpha, k} & =\sum_{i=1}^{p-1} \sum_{j=1}^{i+1} \xi_{i, j} E_{r k} E_{i j}-\sum_{j=0}^{k-1} \sum_{i=1}^{p-j} \xi_{k, k-j} E_{r k} E_{i+j, i} \\
& =\sum_{j=1}^{k+1} \xi_{k, j} E_{r j}-\sum_{j=1}^{k} \xi_{k, j} E_{r j} \\
& =\xi_{k, k+1} E_{r, k+1} .
\end{aligned}
$$

Equality $\xi_{k, k+1}=k h(\alpha)$ concludes the proof.

\section{Proposition 4.7.}

(a) Assume $\alpha \in Z(\mathrm{~B})$. Then $\varepsilon_{\alpha}\left(\mathrm{A}_{h}(\mathrm{~B})\right)$ contains $h(\alpha)^{2(p-1)} M_{p}(\mathrm{~B})$.

(b) Assume $h(\alpha)$ is invertible in $\mathrm{B}$ for some $\alpha \in Z(\mathrm{~B})$. Then $\operatorname{Id}\left(\mathrm{A}_{h}(\mathrm{~B})\right) \subset$ $\operatorname{Id}\left(M_{p}(\mathrm{~B})\right)$.

(c) Assume $h(\alpha)$ is not a zero divisor for some $\alpha \in Z(\mathrm{~B})$ and $\mathbb{F}$ is infinite. Then $\operatorname{Id}\left(\mathrm{A}_{h}(\mathrm{~B})\right) \subset \operatorname{Id}\left(M_{p}(\mathrm{~B})\right)$.

Proof. For short, we write $\beta$ for $h(\alpha) \in Z(\mathrm{~B})$.

(a) Denote by $\mathcal{L}=\varepsilon_{\alpha}\left(\mathrm{A}_{h}(\mathrm{~B})\right)=\operatorname{alg}_{\mathrm{B}}\left\{I_{p}, C_{\alpha}, D_{\alpha}\right\}$ the $\mathbb{F}$-algebra generated by $\mathrm{B} I_{p}$, $C_{\alpha}, D_{\alpha}$. Since $A_{0}=C_{\alpha}-\alpha I_{p}$, we obtain that

$$
A_{0}^{k}=\sum_{i=1}^{p-k} E_{k+i, i} \in \mathcal{L}
$$

for all $0 \leq k<p$. In particular, $E_{p 1}=A_{0}^{p-1} \in \mathcal{L}$. Equality (8) implies that $D_{\alpha, k} \in \mathcal{L}$ for all $1 \leq k<p$.

The statement of part (a) follows from the following claim:

$$
\left\{\beta^{p+k-r-1} E_{r k} \mid 1 \leq r, k \leq p\right\} \subset \mathcal{L} \text {. }
$$

To prove the claim we use descending induction on $r$.

Assume $r=p$. We have $E_{p 1} \in \mathcal{L}$. Lemma 4.6 implies that $E_{p 1} D_{\alpha, 1}=\beta E_{p 2}$. Since $E_{p 1}, D_{\alpha, 1}$ belong to $\mathcal{L}$, we can see that $\beta E_{p 2} \in \mathcal{L}$. Similarly, the equality $\beta E_{p 2} D_{\alpha, 2}=2 \beta^{2} E_{p 3}$ implies $\beta^{2} E_{p 3} \in \mathcal{L}$. Repeating this reasoning we obtain that $\beta^{k-1} E_{p k} \in \mathcal{L}$ for all $1 \leq k \leq p$.

Assume that for some $1 \leq r<p$ claim (9) holds for all $r^{\prime}>r$, i.e., for every $1 \leq k \leq p$ we have $\beta^{p+k-r^{\prime}-1} E_{r^{\prime} k} \in \mathcal{L}$. Since

$$
\beta^{p-r}\left(A_{0}^{r-1}-\sum_{k=2}^{p-r+1} E_{(r-1)+k, k}\right)=\beta^{p-r} E_{r 1},
$$

we obtain $\beta^{p-r} E_{r 1} \in \mathcal{L}$. Lemma 4.6 implies that $\beta^{p-r} E_{r 1} D_{\alpha, 1}=\beta^{p+1-r} E_{r 2}$. Hence $\beta^{p+1-r} E_{r 2} \in \mathcal{L}$. Repeating this reasoning we obtain that $\beta^{p+k-r-1} E_{r k} \in \mathcal{L}$ 
for all $1<k \leq p$, since $\beta^{p+k-r-2} E_{r, k-1} D_{\alpha, k-1}=(k-1) \beta^{p+k-r-1} E_{r, k}$. Claim (9) is proven.

(b) Since $h(\alpha)$ is invertible in B, part (a) implies that $\varepsilon_{\alpha}\left(\mathrm{A}_{h}(\mathrm{~B})\right)=M_{p}(\mathrm{~B})$. Since $\varepsilon_{\alpha}$ is a homomorphism of $\mathbb{F}$-algebras, the required is proven.

(c) Consider a polynomial identity $f \in \mathbb{F}\left\langle x_{1}, \ldots, x_{m}\right\rangle$ for $\mathrm{A}_{h}(\mathrm{~B})$. Since $\mathbb{F}$ is infinite, without loss of generality we can assume that $f$ is homogeneous with respect to the natural grading of $\mathbb{F}\left\langle x_{1}, \ldots, x_{m}\right\rangle$ by degrees, i.e., each monomial of $f$ has one and the same degree $t>0$. Part (a) implies that for every $A_{1}, \ldots, A_{m}$ from $M_{p}(\mathrm{~B})$ there exist $a_{1}, \ldots, a_{m}$ from $A_{h}(\mathrm{~B})$ such that

$$
\beta^{2(p-1) t} f\left(A_{1}, \ldots, A_{m}\right)=f\left(\beta^{2(p-1)} A_{1}, \ldots, \beta^{2(p-1)} A_{m}\right)=f\left(\varepsilon_{\alpha}\left(a_{1}\right), \ldots, \varepsilon_{\alpha}\left(a_{m}\right)\right) .
$$

Since $\varepsilon_{\alpha}$ is a homomorphism of $\mathbb{F}$-algebras, we have $f\left(\varepsilon_{\alpha}\left(a_{1}\right), \ldots, \varepsilon_{\alpha}\left(a_{m}\right)\right)=0$. Therefore $f$ is a polynomial identity for $\mathrm{A}_{h}(\mathrm{~B})$ because $\beta$ is not a zero divisor.

To illustrate the proof of part (a) of Proposition 4.7 we repeat it in the partial case of $p=3$ in the following example.

Example 4.8. Assume $p=3$ and $h(\alpha) \neq 0$ for some $\alpha \in Z(\mathrm{~B})$. For short, denote $\beta=h(\alpha), \beta^{\prime}=h^{\prime}(\alpha)$ and $\beta^{\prime \prime}=h^{\prime \prime}(\alpha)$. Then $A_{0}=E_{21}+E_{32}$,

$$
C_{\alpha}=\left(\begin{array}{ccc}
\alpha & 0 & 0 \\
1 & \alpha & 0 \\
0 & 1 & \alpha
\end{array}\right), \quad \text { and } \quad D_{\alpha}=\left(\begin{array}{ccc}
\beta^{\prime} & \beta & 0 \\
\beta^{\prime \prime} & 2 \beta^{\prime} & 2 \beta \\
0 & 0 & 0
\end{array}\right) \text {. }
$$

To show that $\mathcal{L}=\operatorname{alg}_{\mathrm{B}}\left\{I_{p}, C_{\alpha}, D_{\alpha}\right\}$ contains $\beta^{4} M_{3}(\mathrm{~B})$, we consider the following elements of $\mathcal{L}$ :

$$
\begin{aligned}
D_{\alpha, 1}=D_{\alpha}-\beta^{\prime} I_{3}= & \left(\begin{array}{ccc}
0 & \beta & 0 \\
\beta^{\prime \prime} & \beta^{\prime} & 2 \beta \\
0 & 0 & -\beta^{\prime}
\end{array}\right) \\
D_{\alpha, 2}=D_{\alpha}-2 \beta^{\prime} I_{3}-\beta^{\prime \prime} A_{0} & =\left(\begin{array}{ccc}
-\beta^{\prime} & \beta & 0 \\
0 & 0 & 2 \beta \\
0 & -\beta^{\prime \prime} & -2 \beta^{\prime}
\end{array}\right) .
\end{aligned}
$$

Note that $A_{0}=C_{\alpha}-\alpha I_{3}$ and $A_{0}^{2}=E_{31}$ belong to $\mathcal{L}$. Since

$$
E_{31} D_{\alpha, 1}=\beta E_{32} \quad \text { and } \quad \beta E_{32} D_{\alpha, 2}=2 \beta^{2} E_{33},
$$

we obtain that $\beta E_{32}, \beta^{2} E_{33} \in \mathcal{L}$. Thus $\beta\left(A_{0}-E_{32}\right)=\beta E_{21}$ lies in $\mathcal{L}$. Since

$$
\beta E_{21} D_{\alpha, 1}=\beta^{2} E_{22} \text { and } \beta^{2} E_{22} D_{\alpha, 2}=2 \beta^{3} E_{23},
$$

we obtain that $\beta^{2} E_{22}, \beta^{3} E_{23} \in \mathcal{L}$. Hence $\beta^{2}\left(I_{3}-E_{22}-E_{33}\right)=\beta^{2} E_{11}$ lies in $\mathcal{L}$. Since

$$
\beta^{2} E_{11} D_{\alpha, 1}=\beta^{3} E_{12} \quad \text { and } \quad \beta^{3} E_{12} D_{\alpha, 2}=2 \beta^{4} E_{13},
$$

we obtain that $\beta^{3} E_{12}, \beta^{4} E_{13} \in \mathcal{L}$. Therefore, $\mathcal{L}$ contains $\beta^{4} M_{3}(\mathrm{~B})$.

Theorem 4.9. Assume that $\mathbb{F}$ is an infinite field of characteristic $p>0$.

(a) If $h(\alpha)$ is not a zero divisor for some $\alpha \in Z(\mathrm{~B})$, then $\mathrm{A}_{h}(\mathrm{~B}) \sim_{\mathrm{PI}} M_{p}(\mathrm{~B})$.

(b) If $\mathrm{B}=\mathbb{F}$, then $\mathrm{A}_{h} \sim_{\mathrm{PI}} M_{p}$. 
Proof. Part (a) follows from Corollary 4.3 and part (c) of Proposition 4.7.

Assume that $\mathrm{B}=\mathbb{F}$. Then there exists $\alpha \in \mathbb{F}$ with $h(\alpha) \neq 0$, because $h \in \mathbb{F}[x]$ is not zero and $\mathbb{F}$ is infinite. Part (a) concludes the proof of part (b).

Corollary 4.10. Assume that $\mathbb{F}$ is an infinite field of characteristic $p>0$ and for $h=\eta_{d} x^{d}+\eta_{d-1} x^{d-1}+\cdots+\eta_{0}$ from $Z(\mathrm{~B})[x]$ we have that $\eta_{d}$ and $\eta_{0}$ are not zero divisors. Then $\mathrm{A}_{h}(\mathrm{~B}) \sim_{\mathrm{PI}} M_{p}(\mathrm{~B})$.

\section{5. $A_{h}$ OVER FINITE FIELDS}

In this section we assume that $\mathrm{B}=\mathbb{F}$ is the field of finite of order $q=p^{k}$ and $\mathbb{F} \subset \mathbb{K}$ for an infinite field $\mathbb{K}$. Since $h \in \mathbb{F}[x]$, Convention 1.3 is equivalent to the inequality $h \neq 0$. As above, we write $M_{p}$ for $M_{p}(\mathbb{F})$ and $\mathrm{A}_{h}$ for $\mathrm{A}_{h}(\mathbb{F})$. Given a $\mathbb{K}$-algebra $\mathcal{A}$, we write $\operatorname{Id}_{\mathbb{K}}(\mathcal{A})$ for the ideal of $\mathbb{K}\langle X\rangle$ of polynomial identities for $\mathcal{A}$ over $\mathbb{K}$. In this section we proof the next result.

\section{Theorem 5.1.}

(a) $\operatorname{Id}_{\mathbb{K}}\left(M_{p}(\mathbb{K})\right) \bigcap \mathbb{F}\langle X\rangle \subset \operatorname{Id}\left(\mathrm{A}_{h}\right)$.

(b) $\operatorname{Id}\left(\mathrm{A}_{h}\right) \subset \operatorname{Id}\left(M_{p}\right)$, if $h(\alpha) \neq 0$ for some $\alpha \in \mathbb{F}$.

(c) $\mathrm{A}_{h} \chi_{\mathrm{PI}} M_{p}$.

Proof. Since $A_{h} \subset A_{h}(\mathbb{K})=A_{h} \otimes_{\mathbb{F}} \mathbb{K}$ as $\mathbb{F}$-algebras, we can see that

$$
\operatorname{Id}\left(\mathrm{A}_{h} \otimes_{\mathbb{F}} \mathbb{K}\right)=\operatorname{Id}_{\mathbb{K}}\left(\mathrm{A}_{h}(\mathbb{K})\right) \cap \mathbb{F}\langle X\rangle \subset \operatorname{Id}\left(\mathrm{A}_{h}\right) .
$$

Part (b) of Theorem 4.9 concludes the proof of part (a). Part (b) follows from part (b) of Proposition 4.7.

Consider $F_{p, q}(x, y)=G_{p, q}(x) R_{p, q}(x, y)\left(y^{q}-y\right)$ of $\mathbb{F}\langle x, y\rangle$, where

$$
\begin{aligned}
G_{p, q}(x) & =\left(x^{q^{2}}-x\right)\left(x^{q^{3}}-x\right) \cdots\left(x^{q^{p}}-x\right), \\
R_{p, q}(x, y) & =\left(1-\left(y(\operatorname{ad} x)^{p-1}\right)^{q-1}\right)\left(1-\left(y(\operatorname{ad} x)^{p-2}\right)^{q-1}\right) \cdots\left(1-(y \operatorname{ad} x)^{q-1}\right)
\end{aligned}
$$

for $y \operatorname{ad} x=[y, x]$. Genov [16] proved that $F_{p, q}(x, y)$ is a polynomial identity for $M_{p}$.

Since $x$ ad $x=[x, x]=0$, for $x \in \mathrm{A}_{h}$ we have $R_{p, q}(x, x)=1$ and

$$
F_{p, q}(x, x)=\left(x^{q}-x\right)\left(x^{q^{2}}-x\right)\left(x^{q^{3}}-x\right) \cdots\left(x^{q^{p}}-x\right) .
$$

By part (b) of Lemma 2.5 elements $x, x^{2}, x^{3}, \ldots$ are linearly independent in $\mathrm{A}_{h}$. Therefore, $F_{p, q}(x, x) \neq 0$ in $\mathrm{A}_{h}$; part (c) is proven.

Conjecture 5.2. $\operatorname{Id}\left(M_{p}(\mathbb{K})\right) \bigcap \mathbb{F}\langle X\rangle=\operatorname{Id}\left(\mathrm{A}_{h}\right)$. 


\section{Counterexample}

In this section we consider a counterexample to show that without Convention 1.3 Theorems 3.2 and 4.9 do not hold. Namely, we consider the commutative algebra $\mathrm{B} \simeq \mathbb{F}^{2}$ of double numbers from Example 2.6] i.e., B has an $\mathbb{F}$-basis $\{1, \zeta\}$ with $\zeta^{2}=0$, and set $h=\zeta$. Note that Convention 1.3 does not hold for $h$. Then the statements of Theorems 3.2 and 4.9 are not valid for $A_{\zeta}\left(\mathbb{F}^{2}\right)=A_{h}(B)$ (see Proposition 6.2 below).

Remark 6.1. If Convention 1.3 does not hold for $h$, then Lemmas 2.2 and 2.3 are still valid for $A_{h}(B)$.

Part (b) of Lemma 2.3 together with Remark 6.1 implies that for all $i, j, r, s \geq 0$ we have

$$
x^{i} \widehat{y}^{j} \cdot x^{r} \widehat{y}^{s}=x^{i+r} \widehat{y}^{s+j}+\zeta j r x^{i+r-1} \widehat{y}^{s+j-1}
$$

where we use conventions that $x^{-1}=0$ and $y^{-1}=0$. Then

$$
\left[x^{i} \widehat{y}^{j}, x^{r} \widehat{y}^{s}\right]=\zeta(j r-i s) x^{i+r-1} \widehat{y}^{s+j-1} \text { in } \mathrm{A}_{\zeta}\left(\mathbb{F}^{2}\right)
$$

The unital finite dimensional Grassmann algebra $\mathrm{G}_{k}$ of rank $k$ has an $\mathbb{F}$-basis

$$
\left\{1, e_{i_{1}} \cdots e_{i_{m}} \mid 1 \leq i_{1}<\cdots<i_{m} \leq k\right\}
$$

and satisfies the defining relations $e_{i}^{2}=0$ and $e_{i} e_{j}=-e_{j} e_{i}$ for all $1 \leq i, j \leq k$. The polynomial identities for $\mathrm{G}_{k}$ were described by Di Vincenzo [11] for $p=0$ and by Giambruno, Koshlukov [17 for any infinite field.

Proposition 6.2. Assume that $\mathbb{F}$ is an infinite field.

(a) The T-ideal of identities $\operatorname{Id}\left(\mathrm{A}_{\zeta}\left(\mathbb{F}^{2}\right)\right)$ is generated by

$$
f_{1}=\left[\left[x_{1}, x_{2}\right], x_{3}\right], \quad f_{2}=\left[x_{1}, x_{2}\right]\left[x_{3}, x_{4}\right] .
$$

(b) $\mathrm{A}_{\zeta}\left(\mathbb{F}^{2}\right) \chi_{\mathrm{PI}} M_{t}(\mathrm{C})$ for every $t \geq 2$ and every $\mathbb{F}$-algebra $\mathrm{C}$ with unity.

(c) $\mathrm{A}_{\zeta}\left(\mathbb{F}^{2}\right) \sim_{\mathrm{PI}} \mathrm{G}_{k}$ if and only if $k \in\{2,3\}$.

Proof. (a) By $\mathbb{F}$-linearity formula (11) implies that $[a, b]$ belongs to $\zeta \mathrm{A}_{\zeta}\left(\mathbb{F}^{2}\right)$ for all $a, b \in \mathrm{A}_{\zeta}\left(\mathbb{F}^{2}\right)$. Then $f_{1}, f_{2} \in \mathbb{F}\langle X\rangle$ are nontrivial polynomial identities for $\mathrm{A}_{\zeta}\left(\mathbb{F}^{2}\right)$, since $\zeta^{2}=0$. Note that

$$
f_{3}=\left[x_{1}, x_{2}\right] x_{3}\left[x_{4}, x_{5}\right]=\left[\left[x_{1}, x_{2}\right], x_{3}\right]\left[x_{4}, x_{5}\right]+x_{3}\left[x_{1}, x_{2}\right]\left[x_{4}, x_{5}\right] \in \operatorname{Id}\left(\mathrm{A}_{\zeta}\left(\mathbb{F}^{2}\right)\right)
$$

follows from $f_{1}, f_{2}$. Denote by $I$ the T-ideal generated by $f_{1}, f_{2}$.

Assume that $f=\sum_{k} \alpha_{k} w_{k}$ is a nontrivial identity for $\mathrm{A}_{\zeta}\left(\mathbb{F}^{2}\right)$, where $\alpha_{k} \in \mathbb{F}$ and $w_{k} \in \mathbb{F}\left\langle x_{1}, \ldots, x_{m}\right\rangle$ is a monomial. Since $\mathbb{F}$ is infinite, we can assume that $f$ is multihomogeneous. i.e., there exists $\underline{d} \in \mathbb{N}^{m}$ with $\operatorname{mdeg}\left(w_{k}\right)=\underline{d}$ for each $k$. We apply equalities

$$
\begin{gathered}
u x_{j} x_{i} v=u x_{i} x_{j} v-u\left[x_{i}, x_{j}\right] v, \\
u\left[x_{i}, x_{j}\right] v=\left[x_{i}, x_{j}\right] u v-\left[\left[x_{i}, x_{j}\right], u\right] v,
\end{gathered}
$$

where monomials $u, v$ can be empty and $i<j$, to monomials $\left\{w_{k}\right\}$ and then repeat this procedure. Since $f_{1}, f_{3} \in \operatorname{Id}\left(\mathrm{A}_{\zeta}\left(\mathbb{F}^{2}\right)\right)$, we finally obtain that there exist $g \in I$, $\alpha_{0}, \alpha_{i j} \in \mathbb{F}$ such that

$$
f=g+\alpha_{0} x_{1}^{d_{1}} \cdots x_{m}^{d_{m}}+\sum_{1 \leq i<j \leq m} \alpha_{i j}\left[x_{i}, x_{j}\right] x_{1}^{d_{1}} \cdots x_{i}^{d_{i}-1} \cdots x_{j}^{d_{j}-1} \cdots x_{m}^{d_{m}} \text { in } \mathbb{F}\langle X\rangle .
$$


Since $f(1, \ldots, 1)=g(1, \ldots, 1)+\alpha_{0}$, we obtain that $\alpha_{0}=0$. Consider $i<j$ with $d_{i}, d_{j} \geq 1$. Making substitutions $x_{i} \rightarrow x, x_{j} \rightarrow \widehat{y}, x_{l} \rightarrow 1$ for each $l$ different from $i$ and $j$, we can see that $0=0+\alpha_{i j}[x, \widehat{y}] x^{d_{i}-1} \widehat{y}^{d_{j}-1}$ in $\mathrm{A}_{\zeta}\left(\mathbb{F}^{2}\right)$. Thus $-\alpha_{i j} \zeta x^{d_{i}-1} \widehat{y}^{d_{j}-1}=0$ in $\mathrm{A}_{\zeta}\left(\mathbb{F}^{2}\right)$. Lemma 2.2 together with Remark6.1 implies that $\alpha_{i j}=0$. Therefore, $f=g$ lies in $I$.

(b) Since $\mathbb{F} \subset \mathrm{C}$, every polynomial identity for $M_{t}(\mathrm{C})$ lies in $\operatorname{Id}\left(M_{t}(\mathbb{F})\right)$. By Amitsur-Levitzki Theorem 2] the minimal degree of a polynomial identity for $M_{t}(\mathbb{F})$ is $2 t$. In particular, $f_{1}$ is not an identity for $M_{t}(\mathrm{C})$.

(c) Since $\mathrm{G}_{k}$ is commutative in case $p=2$ or $k=1$, we can assume that $p \neq 2$ and $k \geq 2$. Note that

$$
f_{2}\left(e_{1}, e_{2}, e_{3}, e_{4}\right)=4 e_{1} e_{2} e_{3} e_{4} \neq 0 \text { in } \mathrm{G}_{k} \text { for } k \geq 4 \text {. }
$$

Thus we can assume that $k \in\{2,3\}$. The $\mathrm{T}$-ideal $\operatorname{Id}\left(\mathrm{G}_{k}\right)$ is generated by

- $f_{1}, f_{2}$ in case $p=0$ or $p=k=3$.

- $f_{1}, \mathrm{St}_{4}$ in case $p>k$, where $k \in\{2,3\}$.

Since

$$
\mathrm{St}_{4}\left(x_{1}, x_{2}, x_{3}, x_{4}\right)=\left[x_{1}, x_{2}\right] \circ\left[x_{3}, x_{4}\right]-\left[x_{1}, x_{3}\right] \circ\left[x_{2}, x_{4}\right]+\left[x_{1}, x_{4}\right] \circ\left[x_{2}, x_{3}\right],
$$

where $u \circ v$ stands for $u v+v u$, part (a) implies that $\operatorname{St}_{4}$ lies in $\operatorname{Id}\left(\mathrm{A}_{\zeta}\left(\mathbb{F}^{2}\right)\right)$. On the other hand, we can see that $f_{2}$ is a polynomial identity for $\mathrm{G}_{k}$ when $k \in\{2,3\}$. Part (c) is proven.

\section{REFERENCES}

[1] J. Alev, F. Dumas, Invariants du corps de Weyl sous l'action de groupes finis (French, with English summary), Communications in Algebra 25 (1997), no. 5, 1655-1672.

[2] A.S. Amitsur, J. Levitzki, Minimal identities for algebras, Proc. Amer. Math. Soc. 1 (1950), 449-463.

[3] M. Awami, M. Van den Bergh, and F. Van Oystaeyen, Note on derivations of graded rings and classification of differential polynomial rings, Bull. Soc. Math. Belg. Sér. A 40 (1988), no. 2, 175-183. Deuxième Contact Franco-Belge en Algèbre (Faulx-les-Tombes, 1987).

[4] V.V. Bavula, Generalized Weyl algebras and their representations (Russian), Algebra i Analiz 4 (1992), no. 1, 75-97. English translation: St. Petersburg Math. J. 4 (1993), no. 1, 71-92.

[5] V.V. Bavula, Classification of simple modules of the Ore extension $K[X]\left[Y ; f \frac{d}{d X}\right]$, Math. Comput. Sci. 14 (2020), 317-325.

[6] V.V. Bavula, Isomorphism problems and groups of automorphisms for Ore extensions K[x]/y; 8] (zero characteristic), arXiv: 2107.09401.

[7] V.V. Bavula, Isomorphism problems and groups of automorphisms for Ore extensions K[x]/y; $f \frac{d}{d x} J$ (prime characteristic), arXiv: 2107.09977.

[8] G. Benkart, S.A. Lopes, M. Ondrus, A parametric family of subalgebras of the Weyl algebra II. Irreducible modules, Recent developments in algebraic and combinatorial aspects of representation theory, 73-98, Contemp. Math., 602, Amer. Math. Soc., Providence, RI, 2013.

[9] G. Benkart, S.A. Lopes, M. Ondrus, A parametric family of subalgebras of the Weyl algebra I. Structure and automorphisms, Transactions of the American Mathematical Society 367 (2015), no. 3, 1993-2021.

[10] G. Benkart, S.A. Lopes, M. Ondrus, Derivations of a parametric family of subalgebras of the Weyl algebra, Journal of Algebra 424 (2015), 46-97.

[11] O.M. Di Vincenzo, A note on the identities of the Grassmann algebras, Unione Matematica Italiana. Bollettino. A. Serie VII, 5 (1991), no. 3, 307-315.

[12] A.S. Dzhumadil'daev, $N$-commutators, Comment. Math. Helv. 79 (2004), no. 3, 516-553.

[13] A.S. Dzhumadil'daev, 2p-commutator on differential operators of order $p$, Lett. Math. Phys. 104 (2014), no. 7, 849-869,

[14] A.S. Dzhumadil'daev, D. Yeliussizov, Path decompositions of digraphs and their applications to Weyl algebra, Adv. in Appl. Math. 67 (2015), 36-54. 
[15] J.A. Freitas, P. Koshlukov, A. Krasilnikov, $\mathbb{Z}$-graded identities of the Lie algebra $W_{1}$, Journal of Algebra 427 (2015), 226-251.

[16] G. Genov, Basis for identities of a third order matrix algebra over a finite field, Algebra Log. 20 (1981), 241-257.

[17] A. Giambruno, P. Koshlukov, On the identities of the Grassmann algebras in characterisitc $p>0$, Israel Journal of Mathematics 122 (2001), 305-316.

[18] A. Giambruno, M. Zaicev, Polynomial identities and asymptotic methods, Math. Surveys Monographs vol. 122, AMS, 2005.

[19] K.R. Goodearl, R.B. Warfield Jr., An Introduction to Noncommutative Noetherian Rings, 2nd edition, Cambridge University Press, 2004.

[20] S.A. Lopes, A. Solotar, Lie structure on the Hochschild cohomology of a family of subalgebras of the Weyl algebra, arXiv: 1903.01226.

[21] S.P. Mishchenko, Solvable subvarieties of a variety generated by a Witt algebra, Math. USSR Sb. 64 (1989), no. 2, 415-426.

[22] Yu. Razmyslov, Identities of algebras and their representations, Transl. Math. Monogr., vol. 138, Amer. Math. Soc., Providence, RI, 1994.

Artem lopatin, State University of Campinas, 651 Sergio Buarque de Holanda, 13083-859 CAmpinas, SP, BraziL

Email address: dr.artem.lopatin@gmail.com (Artem Lopatin)

Carlos Arturo Rodriguez Palma, State University of Campinas, 651 Sergio Buarque de Holanda, 13083-859 Campinas, SP, Brazil; Industrial University of Santander, Cl. 9 \#Cra 27, Ciudad Universitária, Bucaramanga, Santander, Colombia

Email address: carpal1878@gmail.com (Carlos Arturo Rodriguez Palma) 